\title{
Soccer syndrome - Common presentations and manual diagnostic techniques for pelvic malalignment syndrome
}

\author{
Ganesh Elumalai $^{1,}$,, Malarvani Thangamani ${ }^{1}$, Nirmala Palayathan ${ }^{2}$, Ajit Kumar $^{1}$, Manish Kr Singh ${ }^{1}$ \\ ${ }^{1}$ Department of Anatomy, Tribhuvan University, National Medical College, Birgunj, Nepal \\ ${ }^{2}$ Department of Anatomy, Kathmandu University, Nobel Medical College \& Teaching Hospital, Biratnagar, Nepal
}

\section{Email address:}

physiovesalius@gmail.com (E. Ganesh),malardewdrop@gmail.com (T. Malarvani), mailtonirmala.p@gmail.com (P. Nirmala), ajit.anatomy@gmail.com (A. Kumar),manishkrsingh@gmail.com (Manish Kr Singh)

\section{To cite this article:}

Ganesh Elumalai, Malarvani Thangamani, Nirmala Palayathan, Ajit Kumar, Manish Kr Singh. Soccer Syndrome - Common Presentations and Manual Diagnostic Techniques for Pelvic Malalignment Syndrome. American Journal of Sports Science.

Vol. 2, No. 6, 2014, pp. 141-154. doi: 10.11648/j.ajss.20140206.11

\begin{abstract}
The pelvic malalignment syndrome is the most common in sports injuries and even in every client who presents with low back \& pelvis pain or dysfunction. In this study, we used simple bony palpation method to assess the various pelvic malalignments in football players. While there are many methods to determine pelvic symmetry or asymmetry, this method is very simple one and that consistently works well for me. Our method of assessing the pelvic malalignments, even works well to the common individuals suffering with low back \& pelvis pain or dysfunction due to various pelvic malalignment presentation. This study includes the healthy football players from National Football Club (NFC) of our institution, who were training or playing during the period of investigation (September 2012 - September 2014) $(\mathrm{n}=40)$ were eligible for participation. At entry to the study, this eligible sample had a mean age of $22.2 \pm 3.9$ years, height of $175.8 \pm 6.6 \mathrm{~cm}$, and mass of $87.5 \pm 7.1 \mathrm{~kg}$. The number of eligible participants at each time point was 40 for all the four session time, point 1 (T1; start of preseason-1), point 2 (T2; end of season-1), point 3 (T3; start of preseason-2), and point 4 (T4; end of preseason-2). We instructed participants to nominate their leg preference for kicking, which was considered the dominant leg. Among the total number of eligible players assessed, $12.5 \%$ were left-leg dominant, $80 \%$ were right-leg dominant, and $7.5 \%$ reported no leg dominance. Subjects were limited to men who had a normal muscle strength and Range of Motion (ROM) of the back and lower extremities and who had no history of orthopaedic or neurologic disorders. All the individuals are subject to gain access the malalignment of both Innominate and Sacral bones of the bony pelvis unit. In addition, to justify our diagnosis we access the muscles of the functional slings and the muscles around the bony pelvis related to the malalignment of the pelvic unit. The techniques were repeated in different position and also performed for two times per day and continued for fifteen days to standardize. In this study, we observed, $87.5 \%$ i.e., the majority of the soccer's are suffering with multiple pelvic malalignments, includes innominate \& sacral stuck. The soccer's suffering with single component malalignment account for about $10 \%$, includes innominate or sacral stuck and soccer's who had the symmetry pelvis is only $2.5 \%$. Conclusion: This method of assessing the pelvic malalignments until then not documented. In this sense, the objective of this study is to analyze and document the different presentations in pelvic malalignments which are common in soccer players and its simple method of evaluation is the literature state of the art. The present study may provide useful information to analyze common presentations of pelvic malalignments in different sports.
\end{abstract}

Keywords: Pelvic Malalignment, Anterior Pelvic-Tilt, Posterior Pelvic-Tilt, Innominate Upslip

\section{Introduction}

Very rarely both the right and left sides of the body is same. In structural integration we often ignore these side-to-side asymmetries. An understanding of this 'malalignment syndrome' requires the knowledge of common presentations in malalignment and the techniques used to diagnose and treat. This study is mainly focused on the common presentations and manual diagnostic techniques for different pelvic malalignments. We need to learn and customize our assessment to diagnose these structural asymmetries (Liz Gaggini, 2010). This is very important in pelvic asymmetry. 
Because, following the pelvic malalignment, cause the secondary adaptations in the vertebral alignment, leg length, postural and movements modifications to overcome these malalignment.

The pelvic malalignment syndrome is the most common in sports injuries and even in every client who presents with low back \& pelvis pain or dysfunction. An understanding of this 'malalignment syndrome' requires knowledge of the common presentations of malalignment and the techniques used to diagnose and treat. This study is mainly focused on the common presentations and manual diagnostic techniques for pelvic malalignment syndrome. We used simple bony palpation method to assess the various pelvic malalignments in football players. While there are many methods to determine pelvic symmetry or asymmetry, this method is very simple one and that is consistently works well for me. Our method of assessing the pelvic malalignments, even works well to the common individuals suffering with low back \& pelvis pain or dysfunction due to various pelvic malalignment presentation. To be masterly skilled in our current techniques of evaluating the pelvic malalignment it is very simple and essential that you will need to be able to locate the anterior superior iliac spines (ASIS) and posterior superior iliac spines (PSIS), able to judge the levelness of both iliac crests when viewing from the front or rear even in different postures like standing, lying in supine \& prone also while performing the movements during the assessment.

To justify our diagnosis we access the muscles of the functional slings and the muscles around the bony pelvis related to the malalignment of the pelvic unit. The techniques were repeated in different position and also performed for two times per day and continued for fifteen days to standardize.

However, this method of assessing the pelvic malalignments until then not documented. In this sense, the objective of this study to analyze and document the different presentations in pelvic malalignments which are common in soccer players and its simple method of evaluation is the literature state of the art. The present study may provide useful information to analyze common presentations of pelvic malalignments in different sports.

\section{Materials \& Methods}

The healthy football players from National Football Club (NFC) of our institution, who were undergoing training or playing during the period of investigation ( September 2012 - September 2014) $(\mathrm{n}=40)$ were eligible for participation in the study. At entry to the study, this eligible sample had a mean age of $22.2 \pm 3.9$ years, height of $175.8 \pm 6.6 \mathrm{~cm}$, and mass of $87.5 \pm 7.1 \mathrm{~kg}$. The number of eligible participants at each time point was 40 for all the four session time, period 1 (T1; start of preseason - 1), period - 2 (T2; end of season 1), period - 3 (T3; start of preseason - 2), and period - 4 (T4; end of season - 2). We instructed participants to nominate their leg preference for kicking, which was considered the dominant leg. Among the total number of eligible players assessed, $12.5 \%$ were left-leg dominant, $80 \%$ were right-leg dominant, and $7.5 \%$ reported no leg dominance.

Subjects were limited to men who had the normal muscle strength and normal ROM of the back and lower extremities and who had no history of orthopaedic or neurological disorders. All subjects were instructed to restrict excessive physical activity on the day of testing, such as recreational running and bicycling, and to wear gym trunks for the tests. On the day of testing, all subjects reviewed and signed informed consent forms for the study. On assessment we taught the subjects how to perform the movements to gain access to the malalignment of both Innominate and Sacral bones of the bony pelvis unit. In addition, to justify our diagnosis we access the muscles of the functional slings and the muscles around the bony pelvis related to the malalignment of the pelvic unit (Abdolhamid Daneshjoo, 2013), (Julie Hides, 2012), (Fernando Idoate, 2011). We encouraged all the subjects to relax and told them that the movements would not be judged as "good" or "bad." We believed these verbal cues decreased subjects' anxiety and, therefore, helped to standardize the testing. The techniques were repeated in different positions and also performed two times per day and continued for fifteen days to standardize.

\section{Bony Pelvis}

The bony pelvis includes Right \& Left pelvic bones in sides \& front and the Sacrum \& Coccyx behind. They are articulated at Pubic symphysis \& Sacro-Iliac joint (Fig-1). It usually moves as a unit and less often as particular bone. The pelvic malalignments includes, Innominate \& Sacral stuck. The Innominate malalignment involves, Rotational stuck \& Upward stuck. The sacral malalignment includes Nutational stuck, Counter-Nutational stuck \& Rotatory stuck.

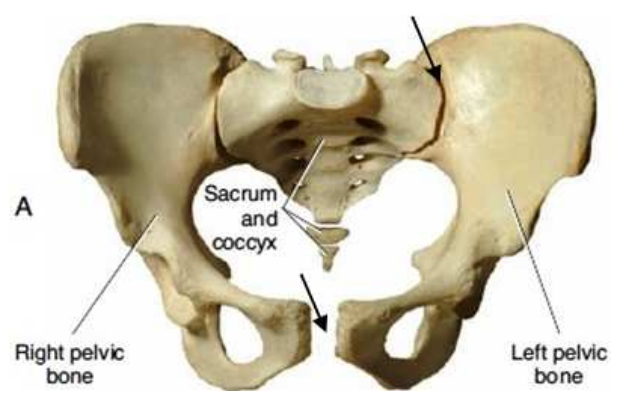

A. Bony Pelvis - Anterior view

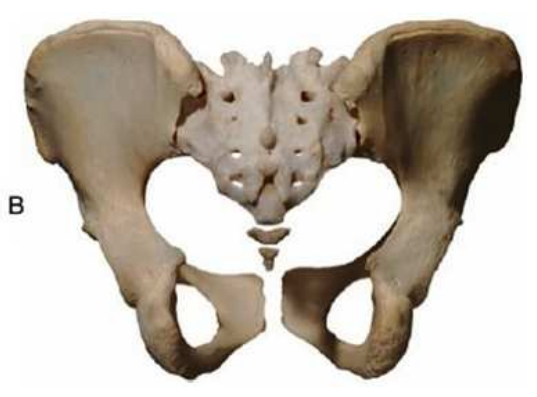

B. Bony Pelvis - Posterior view

Figure 1. Structure of Bony Pelvis 


\section{Movements of Innominate Bone}

1. Rotational movement of innominate possible in 'anterior' or 'posterior' direction, around the coronal axis in the sagittal plane (Fig-2,3,4,5 \& 6). It may occur in one or both innominate and it moves in relative to the sacrum. If both rotates, it may be:

a. In the same direction simultaneously, e.g. as occurs usually on flexion or extension of the trunk or tilting of the pelvis when sitting

Trunk flexion:

Table 1a. Rotatory movements of Innominate during trunk flexion

\begin{tabular}{ll}
\hline Standing (Open Kinematic Chain) & Sitting (Closed Kinematic Chain) \\
\hline Innominates rotates anteriorly & Innominates rotates anteriorly \\
\hline
\end{tabular}

Trunk extension:

Table $1 b$. Rotatory movements of Innominate during trunk extension

\begin{tabular}{ll}
\hline Standing (Open Kinematic Chain) & Sitting (Closed Kinematic Chain) \\
\hline Innominates rotates posteriorly & Innominates rotates posteriorly \\
\hline
\end{tabular}

b. In opposite directions, e.g. as occurs during the normal gait cycle

Right leg in stance \& left leg in swing:

Table 2a. Rotatory movements of Innominate during normal gait cycle

\begin{tabular}{ll}
\hline Right Innominate & Left Innominate \\
\hline Innominate rotates Anteriorly & Innominate rotates posteriorly \\
\hline
\end{tabular}

Left leg in stance \& right leg in swing:

Table $2 \boldsymbol{b}$. Rotatory movements of Innominate during normal gait cycle

\begin{tabular}{ll}
\hline Right Innominate & Left Innominate \\
\hline Innominate rotates posteriorly & Innominate rotates Anteriorly \\
\hline
\end{tabular}

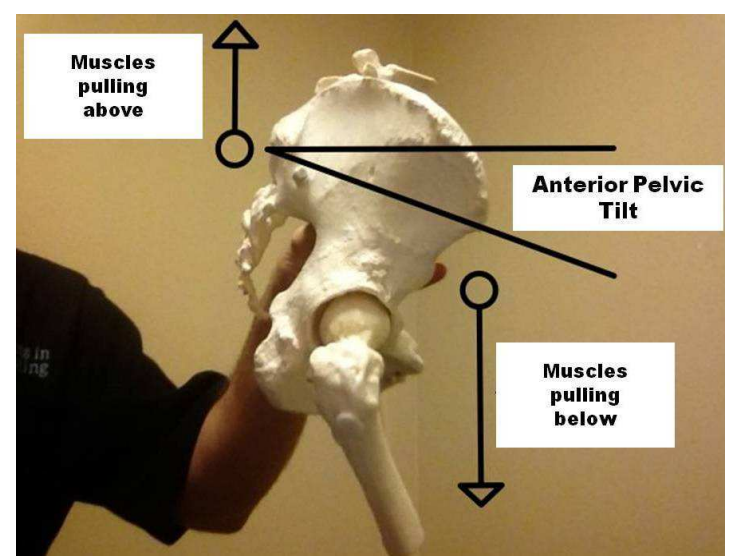

Figure 2. Anterior pelvic tilt of Innominate bone

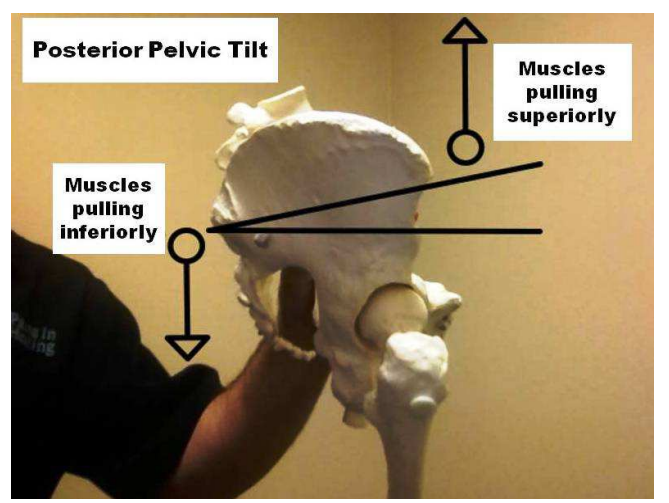

Figure 3. Posterior pelvic tilt of Innominate bone

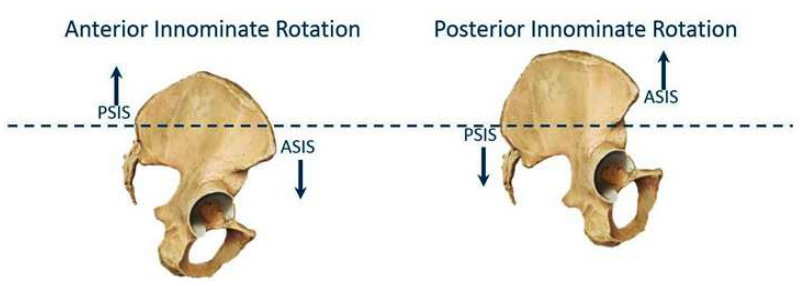

Figure 4. Rotation of Innominate bone

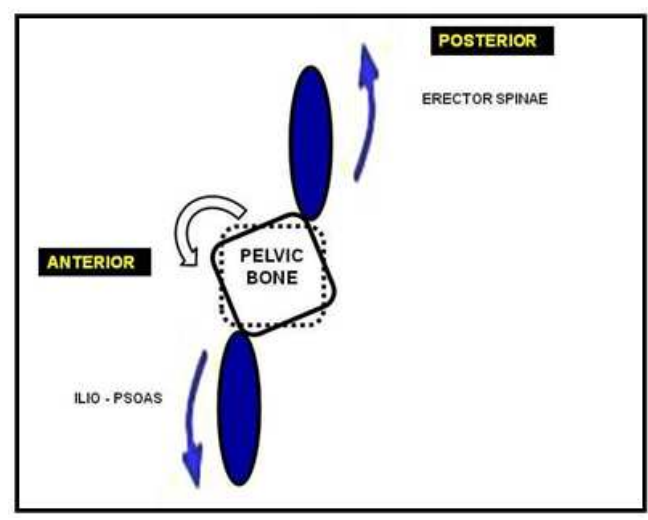

Figure 5. Anterior Rotation Muscles

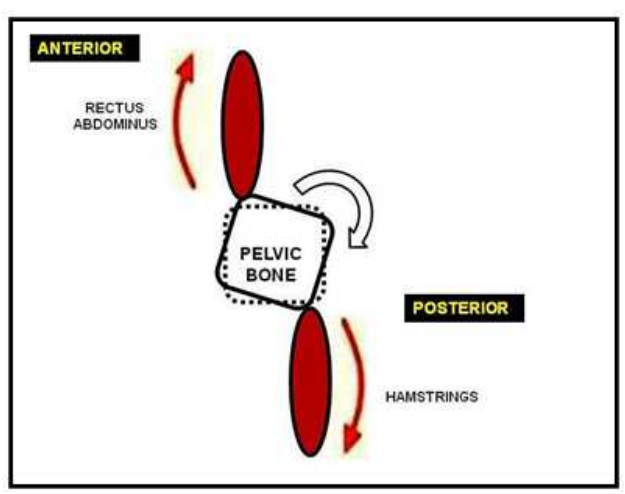

Figure 6. Posterior Rotation Muscles

2. Rotational movement of innominate is also possible as 'Outflare' and 'Inflare'. It refers as movement of the innominates outward and inward, respectively, around the vertical axis in the transverse plane (Fig-7).

It may occur in one or both innominate and it moves in relation to the sacrum. If both rotate, it may be:

a) In the same direction simultaneously, e.g. as occurs 
usually on flexion or extension of the trunk or tilting of the pelvis when sitting

Trunk flexion:

Table 3a. Outflare \& Inflare of Innominate during trunk flexion

\begin{tabular}{ll}
\hline Standing (Open Kinematic Chain) & Sitting (Closed Kinematic Chain) \\
\hline Innominates Outflare & Innominates Outflare \\
\hline
\end{tabular}

Trunk extension:

Table 3b. Outflare \& Inflare of Innominate during trunk extension

Standing (Open Kinematic Chain) $\quad$ Sitting (Closed Kinematic Chain)
Innominates Inflare Innominates Inflare

b. In opposite directions, e.g. as occurs during the normal gait cycle

Right leg in stance \& Left leg in swing:

Table 4a. Outflare \& Inflare of Innominate during normal gait cycle

\begin{tabular}{ll}
\hline Right Innominate & Left Innominate \\
\hline Innominate Outflare & Innominate Inflare \\
\hline
\end{tabular}

Left leg in stance \& Right leg in swing:

Table 4 b. Outflare \& Inflare of Innominate during normal gait cycle

\begin{tabular}{ll}
\hline Right Innominate & Left Innominate \\
\hline Innominate Inflare & Innominate Outflare \\
\hline
\end{tabular}

Figure 7. Inflare and Outflare of Right Innominate bone

\subsection{Muscles responsible to produce "Outflare \& Inflare"}

The Outflare \& Inflare movements are usually associated with the anterior and posterior rotation of innominate bone respectively. The muscles which help to produce the "Outflare \& Inflare" movements are Internal rotators \& External rotators of hip respectively.

3. Innominate "Upslip" (Fig-8 \& 9), is usually associated with superior pubic dysfunction. Normally upslip of unilateral innominate will occur during stair climbing up or down. Missing a step and landing with increased force on one extremity can cause Upslip malalignment of the innominate.

a) With the leg vertical on impact, the force transmitted through the hip joint can result in upward displacement of the innominate relative to the sacrum (a so-called 'upslip').

b) Landing with the leg at a hip-flexion angle on impact results in an 'anterior rotational' force on the innominate which may result in combination with "upslip \& anterior rotation" of innominate.

c) Climbing-up with the leg at a hip-flexion angle on impact results in a 'Posterior rotational' force on the innominate which may result in combination with "upslip \& posterior rotation" of innominate.

\section{INNOMINATE UPSLIP}

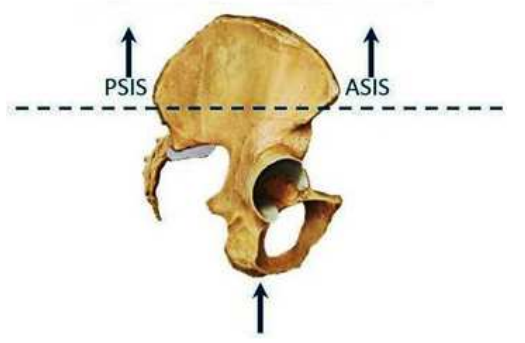

Figure 8. Upslip of Innominate bone

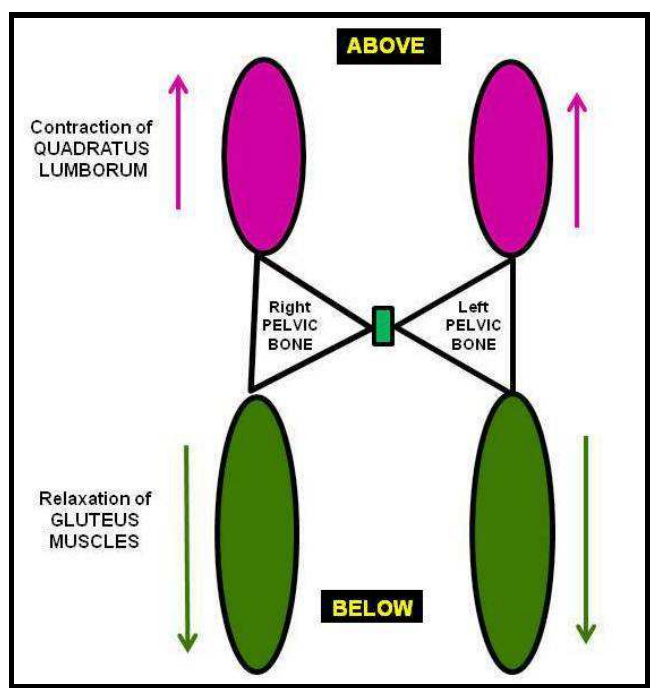

Figure 9. Muscles responsible for Upslip of Innominate bone

\section{Movements of Sacrum}

The sacrum moves at its joints called Sacroiliac \& LumboSacral joints, usually it moves along with the Innominate and / or Lumbar bones in horizontal axis in the vertical plane (Nutation \& Counter-Nutation) (Fig-10,11,13 \& 14) \& Right and Left Oblique axis (Rotation) (Fig-12 \& 15).

Trunk flexion:

Table 5a. Sacral movements during Trunk flexion

\begin{tabular}{|c|c|}
\hline & \\
\hline $\begin{array}{l}\text { - Innominates moves Anteriorly \& } \\
\text { Outflare } \\
\text { - Sacrum undergoes "Nutation" } \\
\text { initially. Once the Interosseus, } \\
\text { Sacrotuberous \& Sacrospinous } \\
\text { ligaments gets tightened, it } \\
\text { undergoes "Counter-Nutation". }\end{array}$ & $\begin{array}{l}\text { inates moves Anteriorly \& } \\
\text { re } \\
\text { n undergoes "Counter- } \\
\text { on". It will cause tightness } \\
\text { ng Dorso-Sacroiliac } \\
\text { nt". Then it results in } \\
\text { or rotation of Innominate }\end{array}$ \\
\hline
\end{tabular}

Trunk extension: 
Table 5b. Sacral movements during Trunk extension

\begin{tabular}{|c|c|}
\hline Standing (Open Kinematic Chain) & Sitting (Closed Kinematic Chain) \\
\hline $\begin{array}{l}\text { - Innominates moves Posteriorly \& } \\
\text { Inflare } \\
\text { - Sacrum produce "Nutation" }\end{array}$ & $\begin{array}{l}\text { Innominates, do not move } \\
\text { initially. } \\
\text { - Sacrum produce "Nutation" } \\
\text { Nutation produce, increased } \\
\text { traction force in Sacrotuberous } \\
\text { ligament below upwards which } \\
\text { cause "anterior rotation' of } \\
\text { innominate. }\end{array}$ \\
\hline
\end{tabular}

Sacral movements during Normal gait cycle

Table 6a. Right leg in stance \& Left leg in swing

Results in

- Right Innominate - moves Anteriorly \& Outflare

- Left Innominate - moves Posteriorly \& Inflare

- Sacrum rotates in Right oblique axis
Table 6b. Left leg in stance \& Right leg in swing

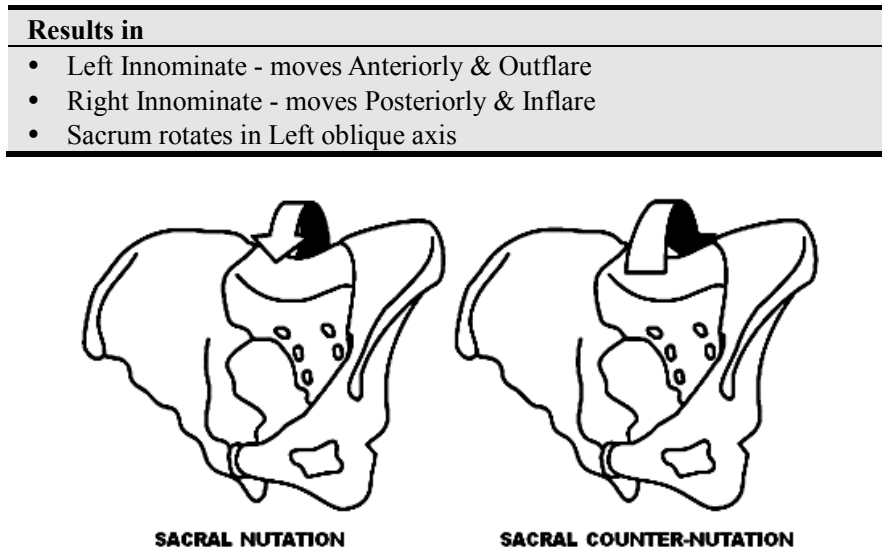

Figure 10. Sacral Nutation and Counter- nutation in relation to the Bony pelvis

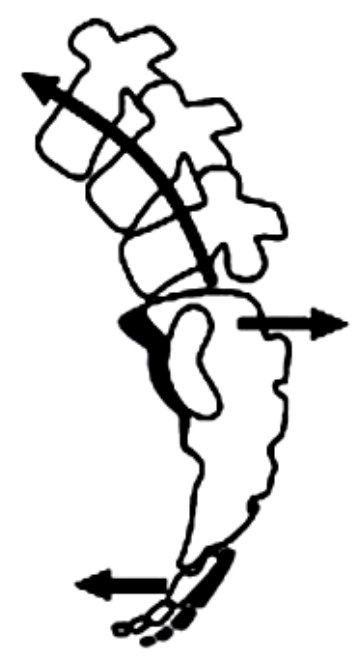
TRUNK FLEXION
(SACRAL COUNTER-NUTATION)

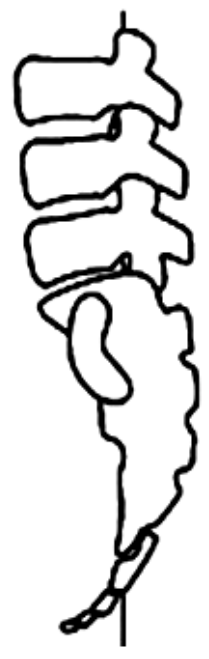

TRUNK NEUTAL

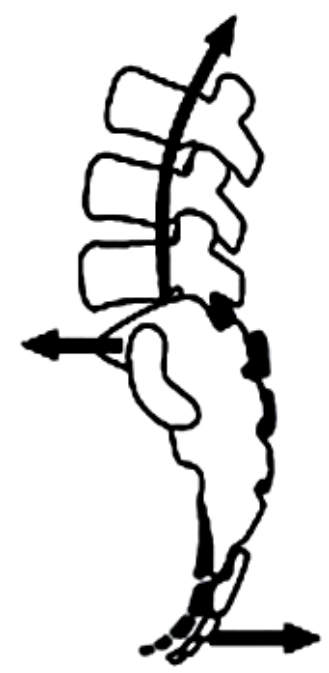

TRUNK EXTENSION
(SACRAL NUTRTION)

Figure 11. Sacral Nutation and Counter- nutation in relation to the Trunk movements

RIGHT LEG IN STANCE \& LEFT LEG IN SWING

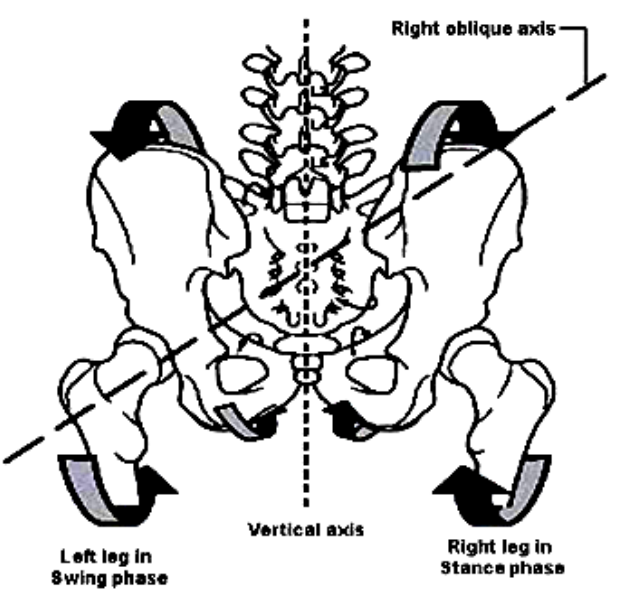

LEFT LEG IN STANCE \& RIGHT LEG IN SWING

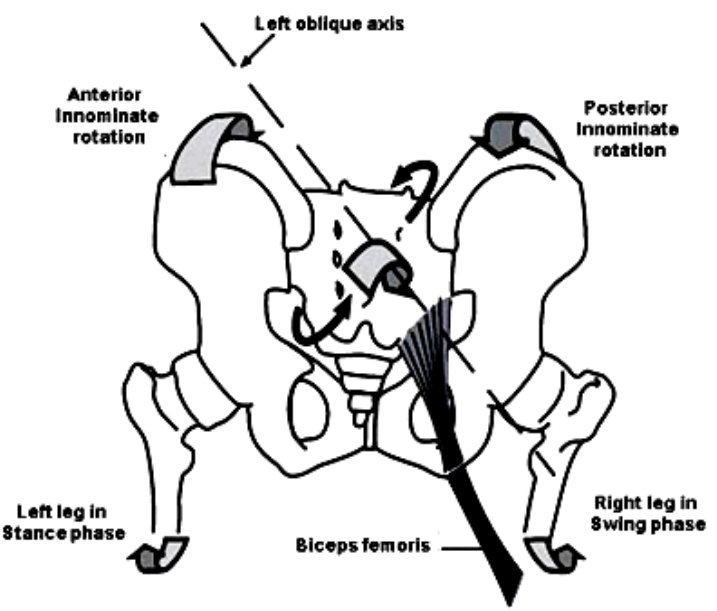

Figure 12. Sacral rotation in oblique axis during Normal Gait cycle 


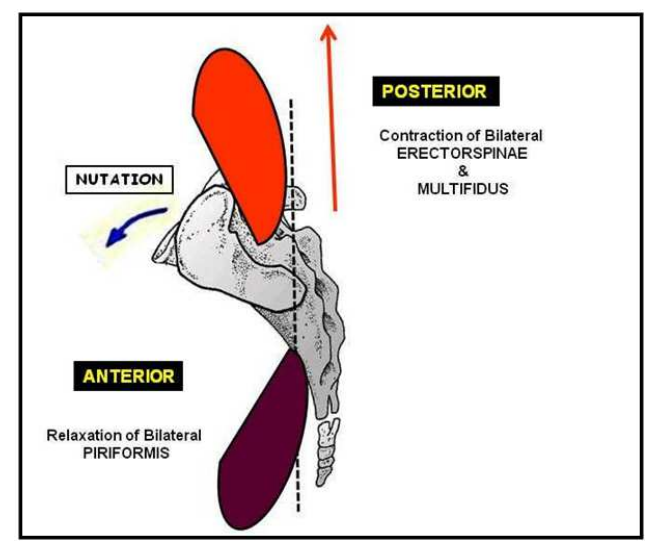

Figure 13. Muscles responsible for nutation of Sacral bone

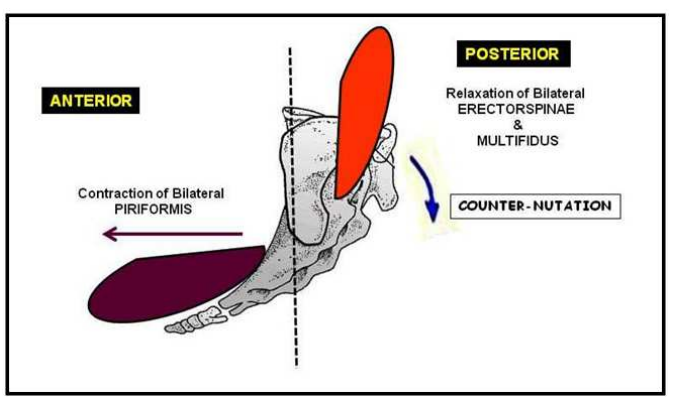

Figure 14. Muscles responsible for Counter - nutation of Sacral bone

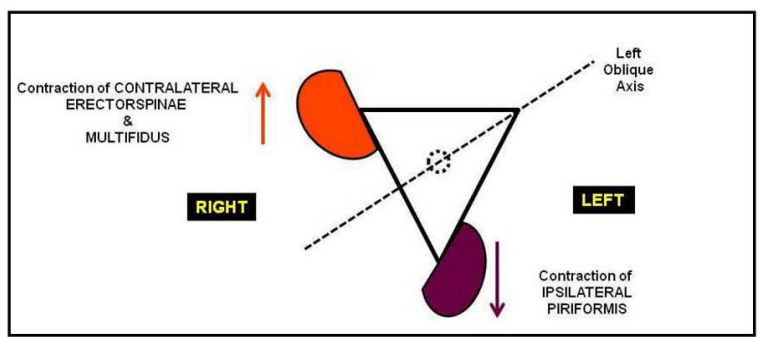

Figure 15. Muscles responsible for Rotation of Sacrum in oblique axis

\section{Stabilizers of the Pelvic Unit}

The 'Stabilizers of the pelvic unit' is made up of oblique and longitudinal systems of 'slings', it is formed by the interconnecting muscles, tendons, ligaments and fascia. The four basic sling systems helps to keep the bony pelvis unit in symmetrical position are:

- Superficial posterior oblique \& longitudinal sling

- Deep posterior oblique \& longitudinal sling

- Anterior oblique \& longitudinal sling

- Lateral longitudinal \& oblique sling

\subsection{Superficial Posterior Oblique \& Longitudinal Sling}

The latissimus dorsi connected, by thoracolumbar fascia, to the contralateral gluteus maximus constitutes the upper part (oblique) of this system. The lower part (longitudinal) of this system is comprised of the continuations of gluteus maximus with the Ilio-Tibial Band (ITB). Concomitant contraction of Tensor-fascia latae and Vastus lateralis helps in further increase effectiveness of ITB.

\subsection{Deep Posterior Oblique \& Longitudinal Sling}

This sling formed by the ipsilateral erector-spinae muscle and contralateral iliocostalis connected (oblique), by way of the deep lamina of the thoracolumbar fascia. The lower part (longitudinal) of this system is comprised of the continuations of iliocostalis with the sacrotuberous ligament and biceps femoris. Concomitant contraction of peroneus longus and tibialis anterior helps $\mathrm{n}$ further increase the effectiveness of this sling.

\subsection{Anterior Oblique \& Longitudinal Sling}

The upper part (oblique) of this sling is formed by external oblique on one side are connected, by way of the anterior abdominal fascia, to the contralateral internal abdominal oblique and the lower part (longitudinal) of this system is comprised of the continuations of internal abdominal oblique with the adductors of the thigh.

\subsection{Lateral Longitudinal \& Oblique Sling}

The upper part (longitudinal) of this sling is formed by the ipsilateral quadratus lumborum from above and the same side gluteus medius, gluteus minimus \& tensor fascia latae from below. The lower part (oblique) of this system is comprised of contralateral adductors of the thigh.

\section{Muscles Responsible to Maintain the Symmetry of Bony Pelvis}

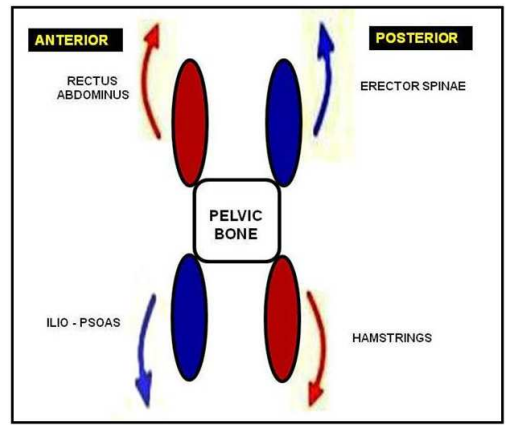

Figure 16. Muscles responsible for symmetry of Pelvic bone

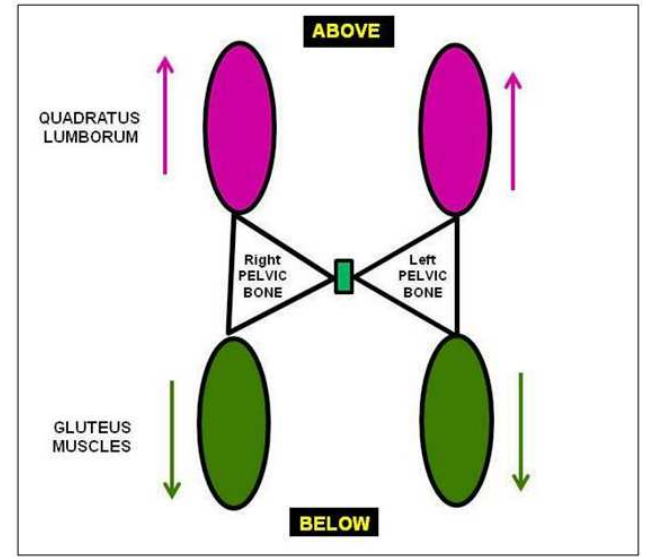

Figure 17. Muscles responsible for lateral stability of Pelvic bone 


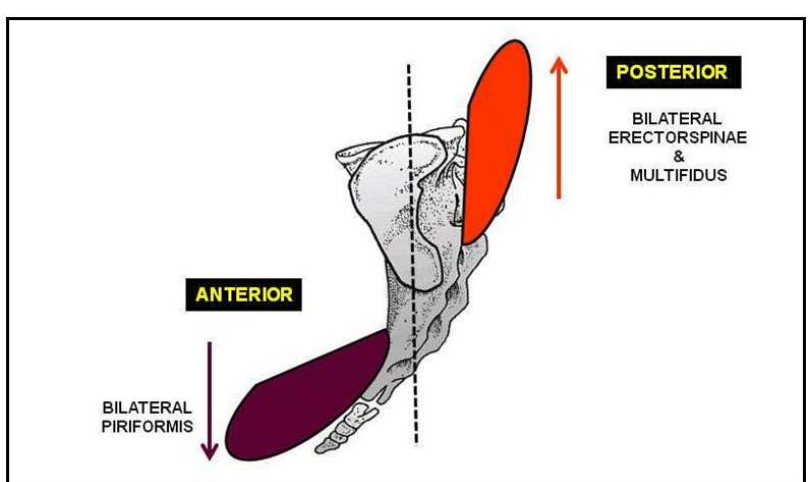

Figure 18. Muscles responsible for symmetry of Sacral bone in Transverse Axis

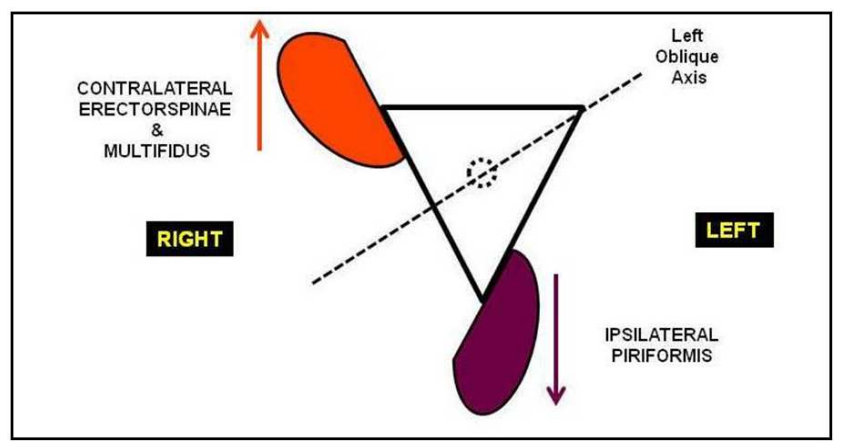

Figure 19. Muscles responsible for symmetry of Sacral bone in Oblique axis

\section{Malalignments in Innominate Bone}

The common presentation may appear in isolation or in combination with one or both of the others. For example, an 'upslip' appears on its own in about $10 \%$, in combination with either 'rotational malalignment' or 'flare' or both in another $10 \%$, for a total of $20 \%$ overall. In this study, three common malalignment presentations are observed in 80 $95 \%$ of individuals. These presentations are:

1. 'rotational malalignment' (80-85\%)

2. pelvic 'flare' - innominate 'outflare' or 'inflare' (40 $50 \%$ ), and the

3. 'upslip' (15-20\%)

The common 'Innominate malalignment' refers to fixation of an innominate bone relative to the sacrum in excessive anterior or posterior rotation in the sagittal plane. Such rotation can affect an innominate on one side only but is more likely to be seen in association with:

1. Compensatory rotation of the contralateral innominate around the coronal axis in the sagittal plane.(e.g. Right side anterior rotation with Left side Posterior rotation)

2. Compensatory rotation of the contralateral innominate around the vertical axis in the transverse plane. (e.g. Right side anterior rotation with outflare \& Left side Posterior rotation with inflare)

3. Upslip of innominate with the displacement of the pubic bone superiorly, relative to each other.

\section{Malalignments in Sacrum}

The sacral malalignment is usually accompanied with malalignment of Innominate and / or Lumbar vertebra. The common sacral malalignment is rotational (oblique axis) stuck, it is usually associated with rotational malalignment of innominate bone.

1. If left Innominate stuck posteriorly \& inflare, the right Innominate undergoes compensatory rotational stuck in anteriorly \& outflare and Sacrum undergoes rotatory stuck in Right oblique axis.

2. If right Innominate stuck posteriorly \& inflare, the left Innominate undergoes compensatory rotational stuck in anteriorly \& outflare and Sacrum undergoes rotatory stuck in Left oblique axis.

3. The bilateral innominate tilt is usually associated with the sacrum stuck in either Nutation or Counter-Nutation.

\section{Assessment of Malalignment}

The initial step in the diagnosis of malalignment is to establish whether asymmetry is present and, if so, it is caused by one (or combination) of the following:

1. an anatomical (true) leg length difference (LLD)

2. presence of True or Compensated Trendelenburg's sign

3. one of the three common presentations of Innominate malalignment, with a functional LLD (seen with an 'upslip' and 'rotational malalignment')

4. sacral stuck

Examination is preferably carried out on a firm, even surface in different postures like Standing, Sitting and Lying. Examination performed on a soft or sagging support, or across a break in the surface (a feature common to medical plinths), may affect the assessment and lead to incorrect conclusions and possibly misdiagnosis. To correct the pelvic malalignment, determination of the type of pelvic malalignment present is the utmost importance.

\subsection{Measurement of Limb Length Discrepancies: (Lying-Sitting-Lying Test)}

This test affords an individual and those caring for patients a quick way of establishing whether malalignment is actually present and, if so, to find out whether there is a 'rotational malalignment', 'upslip', 'flare' or a combination of these, so that appropriate treatment can be initiated.

The measurement of leg length is much easier and more accurate, by comparing the level of the thumbs placed in the hollow sulcus immediately below the medial malleolus on each side, directly overlying the medial ankle ligaments. Point the tip of each thumb straight downward (distal phalanx vertical) helps to compare the relative level of interphalangeal joints (i.e. knuckles) which end up closer together and are more clearly demarcated than the tip malleoli assessment, also it helps to make side-to-side comparison more accurate.

Remember to hold onto the ankles lightly - the thumbs are only serving you as a guide to compare side-to-side leg movement and length on 
sitting and lying. A common mistake is to hold on forcefully, at the risk of impairing free upward and downward movement of the legs, making the person to actual discomfort.

The person initially lies supine and is then asked to sit up. A shift of the pelvis or other error is less likely avoided (to prevent the activation of leg, pelvic or trunk muscles that can influence movement of the pelvis). Then the assessment is repeated with the supine lying, for comparison.

Clinical Correlations:

1 when one leg is shorter by an equal amount in both sitting-up and lying-down

a. 'true' LLD, with all the landmarks are aligned

b. an 'upslip', with all the landmarks, both anterior and posterior, have moved upward on the side of the 'upslip'.

1 when leg length is equal

a. pelvis is in alignment, with all the landmarks symmetrical

b. presence of an 'outflare or inflare' malalignment

2 when leg length shifts

a. suspect a 'rotational malalignment', with landmarks all asymmetrical. But,

b. rule out the person is not sitting or lying slightly asymmetrically with a wallet or other object in a back pocket or on account of a break in the plinth.

\subsection{Assessment of True or Compensated Trendelenburg's (Gait) Sign}

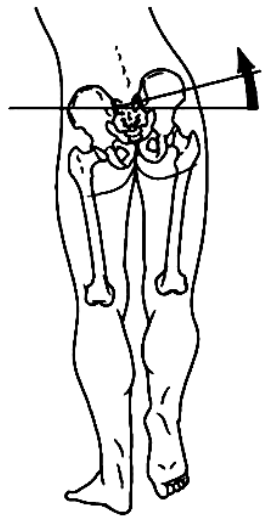

Compensated Trendelenburg's gait
(e.g. Left hip Paralysis)

Figure 20. True \& Compensated Trendelenburg's gait

Asymmetrical weight-bearing plays an important factor on account of an anatomical (true) or a functional leg length difference, attempting the pelvic girdle to compensate for problems to transfer load through the lumbo pelvic- hip complex in order to overcome the insufficiency. It may be either by two ways

\section{a) Compensated Trendelenburg (sign) gait}

If a person is with the weakness in the left hip abductors, it makes him difficult for stabilizing the left hip and SI joint for proper load transfer through the hip, SI joint and up through the lumbo-sacral junction (Fig-20).
When walking, the person compensates by leaning the trunk into the impaired left side during mid-stance, moving the centre of gravity outward from midline and more directly over top of the hip joint, thereby

- decreasing the need for left abductor muscle action to achieve stability of the left hip joint

- decreasing vertical shear forces through the left SI joint. b) True Trendelenburg (sign) gait

A person with the left hip instability caused by the degeneration (osteoarthritis) of the joint or by inflamed and painful joint will have difficulty in stabilizing the left hip and SI joint (Fig-20).

The person may compensate for the impaired ability to transfer load through the left hip by leaning away from that side (adducting the pelvis, abducting the left femur) in midstance, thereby

- bringing the centre of gravity closer to midline (away from the left hip joint and toward the SI joint)

- decreasing stress on the painful left hip joint

- depending more on the strong left hip abductors to ensure stability of the left hip joint and also of the pelvic unit.

\subsection{Assessment of Pelvic Bone Alignment}

It is essential to locate the anterior superior iliac spines (ASIS) and posterior superior iliac spines (PSIS) (Pelvic evaluation, 2010/09), to judge the alignment of pelvic girdle on observing the pelvis from both the front and rear. ASIS level is best judged by kneeling or standing in front of the standing or lying client and hooking your thumbs just under the ASIS, rather than trying to place the thumb on the apex of the ASIS (Fig-21). Mark the level of ASIS on both the side using marker to note the side of high or low. PSIS's are palpated best by kneeling or standing behind the standing or lying client, placing the hands so that the sides of the first finger is resting on the iliac crest (Fig-22) (palms facing down with the thumbs oriented slightly downward). While tracing on the Iliac crest for PSIS palpation, the tips of the thumbs rest on the skin over the PSIS called dimple (of Venus) (Fig-23); look for these first. On other way, you can palpate the PSIS by hooking your thumbs just caudal to the PSIS protuberance. Accurate palpation of the ASIS and PSIS is a matter of practice, accuracy will improve with practice. It tends to be more difficult to locate landmarks with heavier clients and with clients who are very tight or have the high muscle mass.

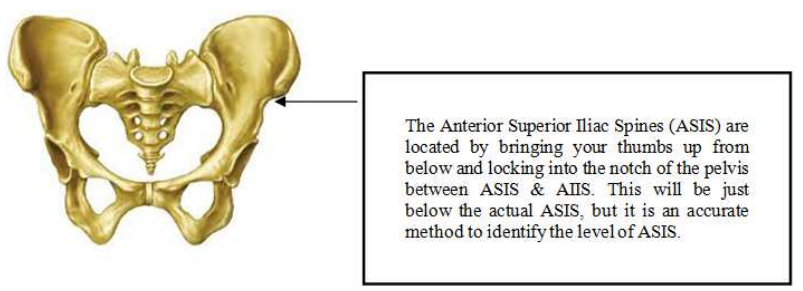

Figure 21. Palpation of Anterior superior iliac spine 


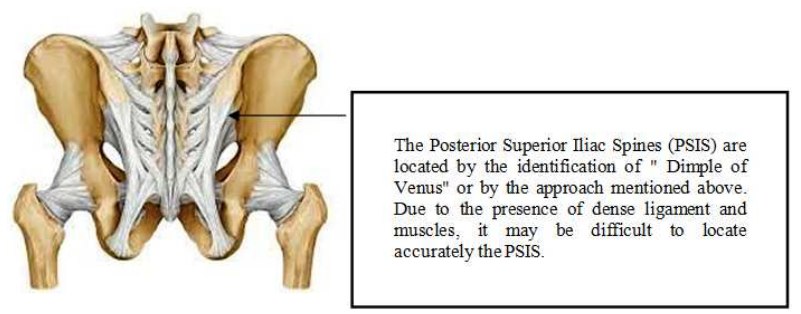

Figure 22. Palpation of Posterior superior iliac spine

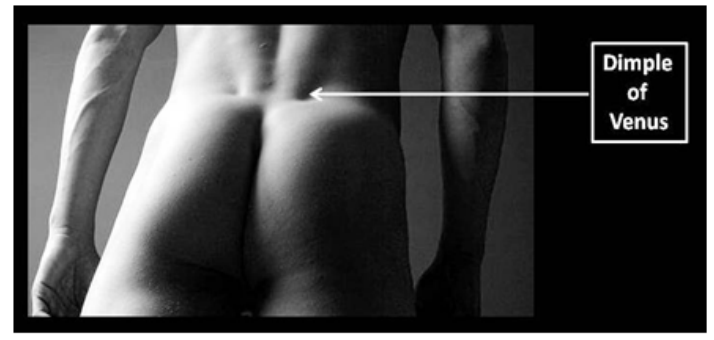

Figure 23. Surface landmark for Posterior superior iliac spine

The level of pubic bone on both the side is best assessed with the patient in supine position, rather than standing or sitting. Subjects are advised to relax the abdominal muscles, the examiner approach the pubis by giving gentle palpation using both the thumbs over the pubic symphysis to assess the level of body of both the pubis.

Clinical Correlations:

The general guidelines on innominate findings: (in relation to the Right innominate)

1 Low right ASIS, high right PSIS, near level Intercristal line:

Anterior rotation of the right innominate or posterior rotation of the left innominate

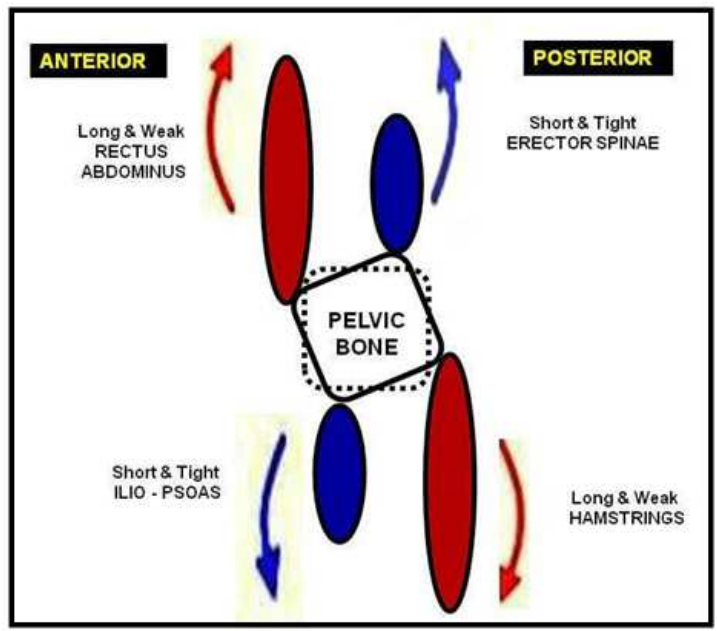

Figure 24. Muscles involved in Anterior Innominate stuck

2 High right ASIS, low right PSIS, near level Intercristal line:

Posterior rotation of the right innominate or anterior rotation of the left innominate

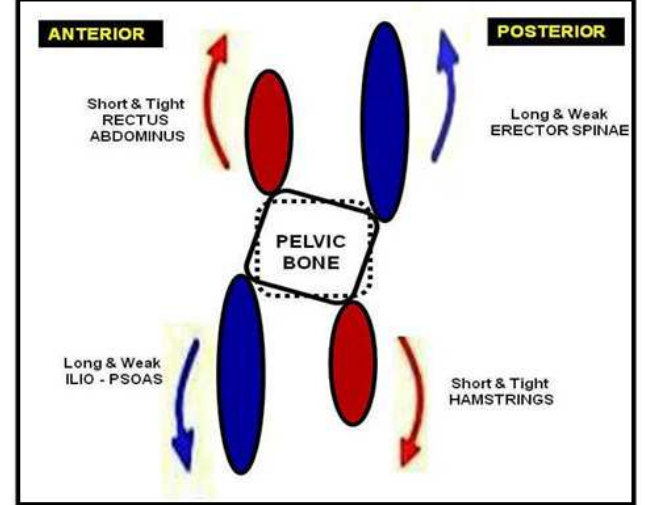

Figure 25. Muscles involved in Posterior Innominate stuck

3 High right ASIS \& PSIS, with right superior pubic: Right innominate up-slip with supra-pubic dysfunction

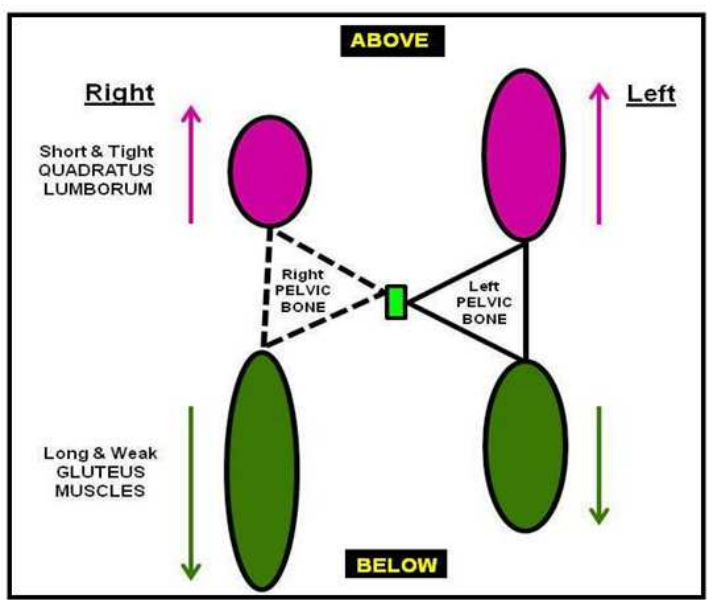

Figure 26. Muscles involved in Right Innominate Upslip stuck

4 Level ASIS's with high right PSIS and high right iliac crest:

Right innominate anterior rotation with a right up-slip

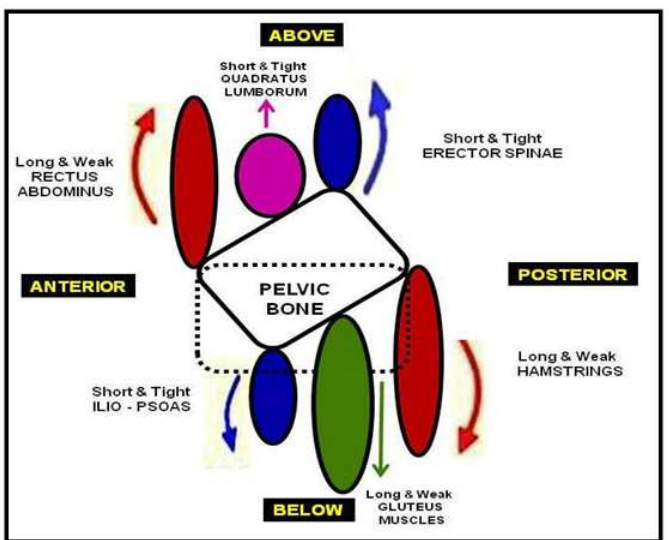

Figure 27. Muscles involved in Right Innominate Upslip \& Anterior rotational stuck

5 High right ASIS with level PSIS's and high right crest: Right innominate posterior rotation with up-slip 


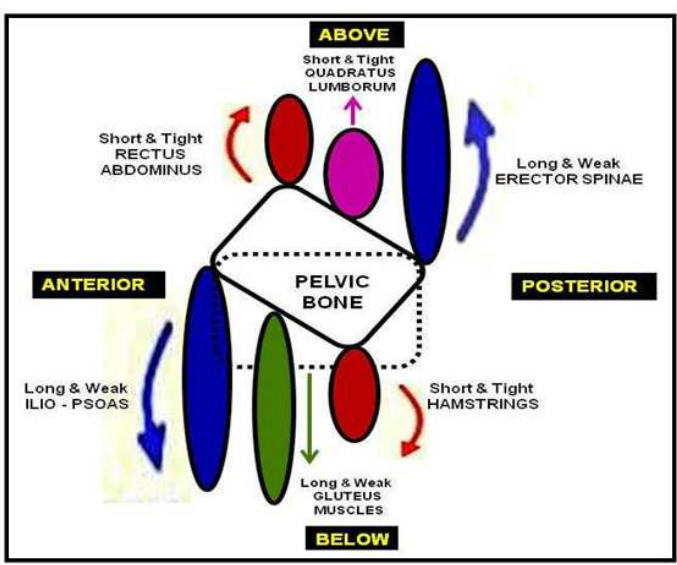

Figure 28. Muscles involved in Right Innominate Upslip \& Posterior rotational stuck

\section{Medial right ASIS with Lateral right PSIS}

Right Inflare innominate

7 Lateral right ASIS with Medial right PSIS

Right Outflare innominate

The above mentioned presentation may appear in isolation or in combination with one or both of the others. The foreseen seven possibilities are applicable also in the left side innominate bone also.

\subsection{Assessment of Sacral Alignment}

It is essential to locate the posterior superior iliac spines (PSIS), to judge the alignment of sacrum. The other finest method to assess the sacral alignment is to identify the position of the sacral base as judged by the sacral sulci. The sulci are formed by the junction of the ala ('wings') of the sacral base with the ilium on either side. Locate the depression on each side at the junction of L5 and S1with the tip of an index finger and then run the fingers outward at this level until they meet the medial edge of the posterior iliac crest (approximately $1.5-2.5 \mathrm{~cm}$ lateral to the midline, often clearly demarcated by an overlying dimple). The true depth of the sulcus is approximately $1.0-1.5 \mathrm{~cm}$, usually reduced to about $0.5-1.0 \mathrm{~cm}$ by the overlying skin and subcutaneous tissues. The depth of the right sulcus should be equal to that on the left and lie in same level in the coronal (frontal) plane.

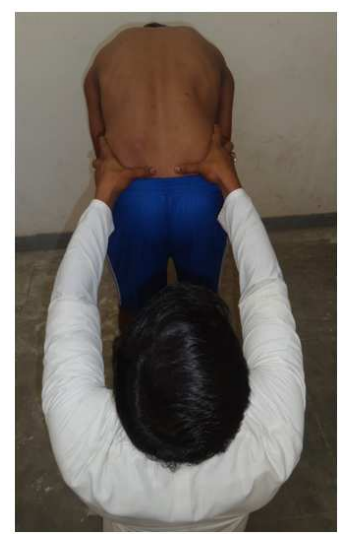

Picture 1. Palpation of Sacral Base
The sacral malalignment can be assessed by the following methods,

1 Standing trunk flexion test: (Wedel, 2013)

Sacrum undergoes "Nutation" initially. Once the Interosseus, Sacrotuberous \& Sacrospinous ligaments gets tightened, it undergoes "Counter-Nutation".

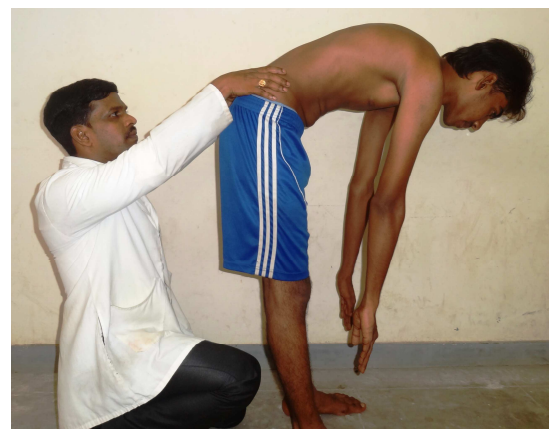

Picture 2. Standing trunkflexion test

2 Sitting trunk flexion test: (Wedel, 2013)

Sacrum undergoes "Counter-Nutation" initially and it will cause tightness in "Long Dorso-Sacroiliac ligament". Then posterior rotation of Innominate occurs along with Nutation of Sacrum.

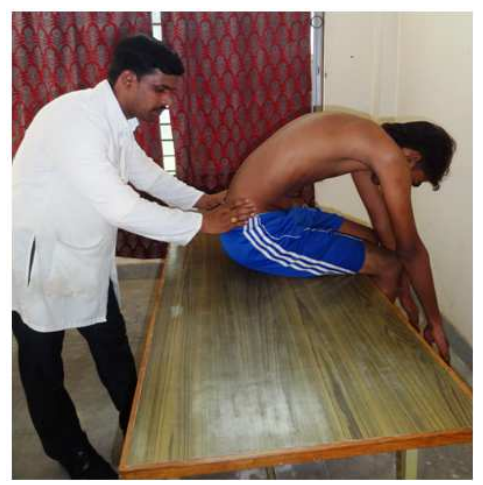

Picture 3. Sitting trunk flexion test

3 Standing single leg flexion test:

This is to assess the rotational stuck of sacrum in oblique axis.

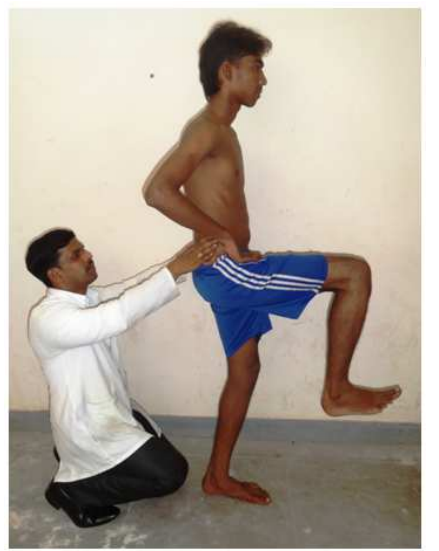

Picture 4. Standing single leg flexion test 


\section{Clinical Correlations:}

The general guidelines on Sacral findings:

1. Both Right \& Left Sacral sulci stuck anteriorly and fails to come back to neutral position during trunk extension from end flexion.

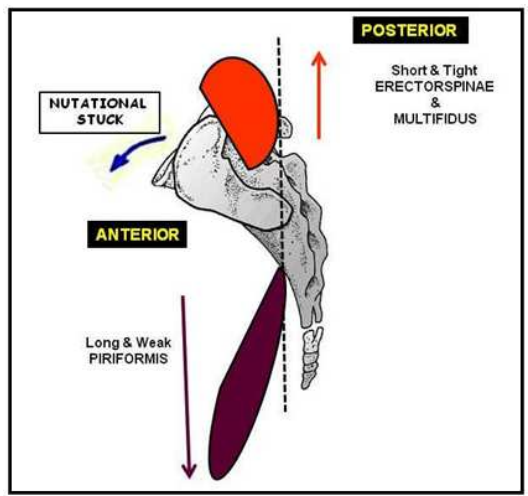

Figure 29. Muscles involved in Nutational stuck of Sacrum

2 Both Right \& Left Sacral sulci stuck posteriorly and fails to come front to neutral position during trunk flexion from end extension.

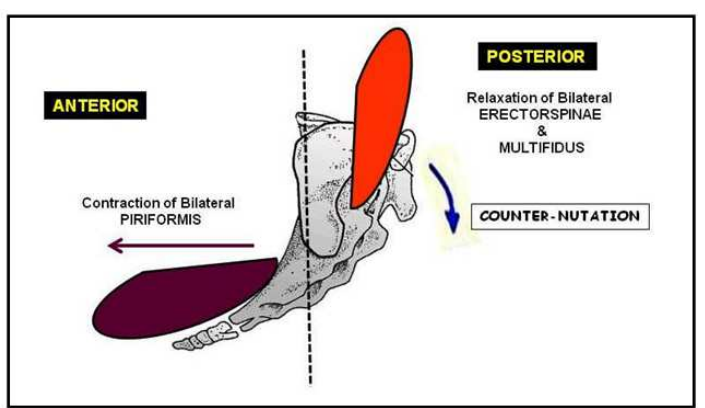

Figure 30. Muscles involved in Counter - Nutational stuck of Sacrum

3. Increase in the depth of Right Sacral sulci than Left and fails to come back to neutral position during hip extension from mid - flexion.

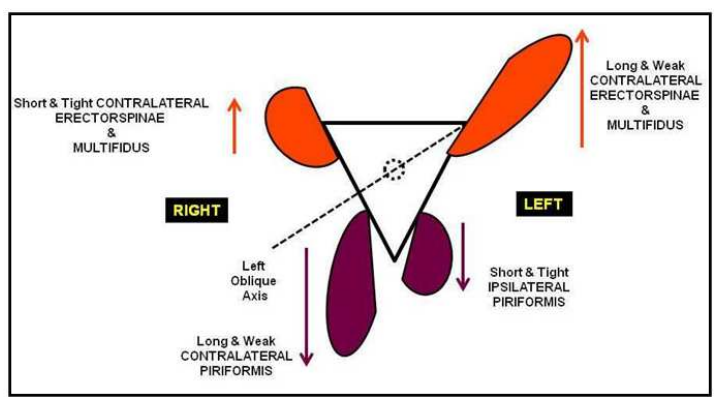

Figure 31. Muscles involved in Left oblique stuck of Sacrum

\subsection{Ganesh et al - Bony Pelvis Malalignment Assessment Chart}

A. Name:

B. Age:

C. Sex:

D. Profession:

E. Complaint(S):

F. Observation(S):

1. Trendelenburg's Sign:

2. leg length:

Table 7. Assessment of Leg length

\begin{tabular}{lllll}
\hline Position & Side & $\begin{array}{l}\text { Increase / } \\
\text { Decrease }\end{array}$ & Side & $\begin{array}{l}\text { Increase / } \\
\text { Decrease }\end{array}$ \\
\hline Long Sitting & Right & & Left & \\
Supine Lying & Right & Left & \\
Long Sitting & Right & Left & \\
\hline
\end{tabular}

3. Innominate Bone Assessment(S)

1. ASIS:

Table 8. Assessment of level of ASIS

\begin{tabular}{lllllllll}
\hline \multicolumn{1}{l}{ Standing } & \multicolumn{8}{c}{ Supine } \\
\hline S & I & M & L & S & I & M & L \\
\hline
\end{tabular}

*S=Superior: $\mathrm{I}=$ Inferior: $\mathrm{M}=$ Medial: $\mathrm{L}=$ Lateral

2. Pubic Symphysis:

Table 9. Assessment of level pubic Symphysis

\begin{tabular}{lll}
\hline \multirow{2}{*}{ Normal } & \multicolumn{2}{l}{ Superior } \\
\cline { 2 - 3 } & Right & Left \\
\hline
\end{tabular}

3. Intercristal Line

Table 10. Assessment of level of Intercristal line

\begin{tabular}{|c|c|c|c|c|}
\hline \multirow{2}{*}{ Neutral } & \multicolumn{2}{|c|}{ Higher } & \multicolumn{2}{|c|}{ Lower } \\
\hline & Right & Left & Right & Left \\
\hline
\end{tabular}

4. PSIS:

Table 11. Assessment of level of PSIS

\begin{tabular}{llllllll}
\hline \multicolumn{1}{l}{ Standing } & \multicolumn{7}{c}{ Supine } \\
\hline S & I & M & L & S & I & M & L \\
\hline
\end{tabular}

*S=Superior: $\mathrm{I}=$ Inferior: $\mathrm{M}=$ Medial: $\mathrm{L}=$ Lateral 


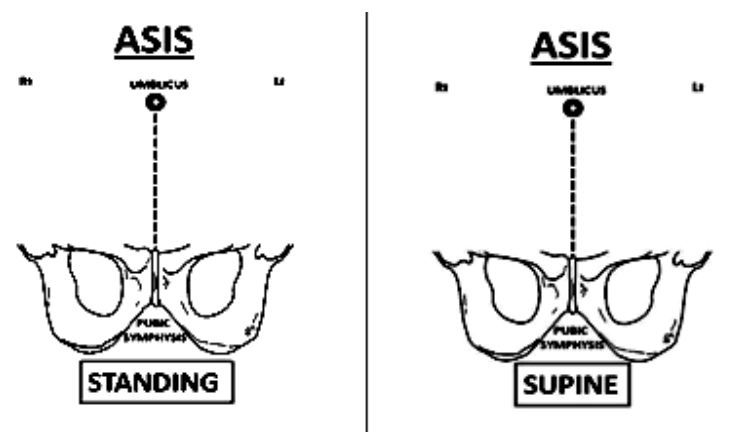

Figure 32. Assessment of the level of ASIS
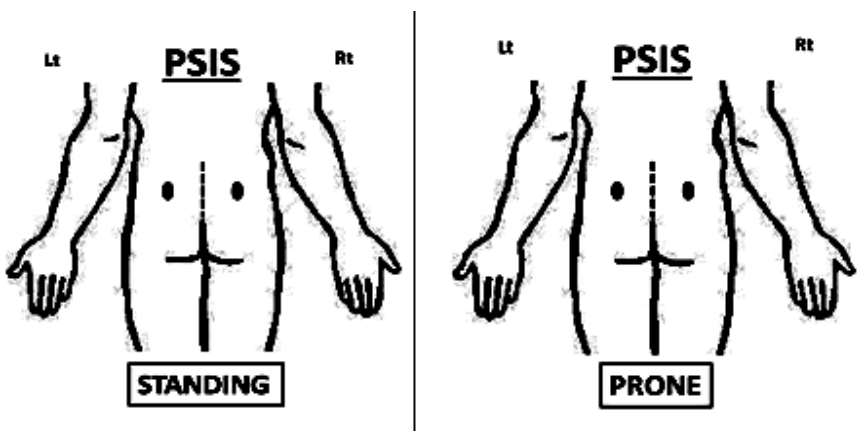

Figure 33. Assessment of the level of PSIS

4. Sacral Assessment:

Table 12. Assessment of Sacral stuck

\begin{tabular}{llllll}
\hline \multicolumn{2}{l}{ Nutation (Forward Bending) } & & Counter Nutation (Back to Normal) & & Rotation (Standing Leg Flexion) \\
\hline Right & Left & Right & Left & Right & Left \\
\hline
\end{tabular}

5. Muscle Assessment:

Table 13. Assessment of Muscle strength responsible for pelvic stability

\begin{tabular}{llll}
\hline \multirow{2}{*}{ Muscle Group \& Its Test } & Muscles & Shortened / Tightened & Lengthened / Weakened \\
\cline { 2 - 3 } & Right & \\
\hline \multirow{2}{*}{ Hip Flexors (Modified Thomas Test) } & Iliopsoas & \\
Hip Extensors & Rectus Femoris & \\
(Reverse Slr) & Gluteus Maximus & \\
Hip Adductors & Hamstrings & \\
& Adductor Longus & \\
Hip Abductors & Gracilis & \\
& Gluteus Medius & \\
Trunk Flexors & Tensor Fascia Latae (TFL) & \\
& Rectus Abdominis & \\
Trunk Extensors & External Oblique & \\
Trunk Side Flexors & Erector Spinae & \\
Sacral Flexors & Multifidus & \\
\hline
\end{tabular}

G. Provisional Diagnosis:

\section{Observations}

Table 14. Period - 1 (Start of preseason - 1)

\begin{tabular}{ll}
\hline Malalignment & Percentage (\%) \\
\hline Multiple (31) & 77.5 \\
Single (07) & 17.5 \\
Symmetry Pelvis (02) & 05 \\
\hline
\end{tabular}

Table 15. Period - 2 (End of season - 1)

\begin{tabular}{ll}
\hline Malalignment & Percentage (\%) \\
\hline Multiple (35) & 87.5 \\
Single (04) & 10 \\
Symmetry Pelvis (01) & 02.5 \\
\hline
\end{tabular}

Table 16. Period - 3 (Start of preseason - 2)

\begin{tabular}{ll}
\hline Malalignment & Percentage (\%) \\
\hline Multiple (36) & 90 \\
Single (03) & 7.5 \\
Symmetry Pelvis (01) & 2.5 \\
\hline
\end{tabular}

Table 17. Period - 4 (End of season - 2)

\begin{tabular}{ll}
\hline Malalignment & Percentage (\%) \\
\hline Multiple (38) & 95 \\
Single (02) & 05 \\
Symmetry Pelvis (00) & 00 \\
\hline
\end{tabular}

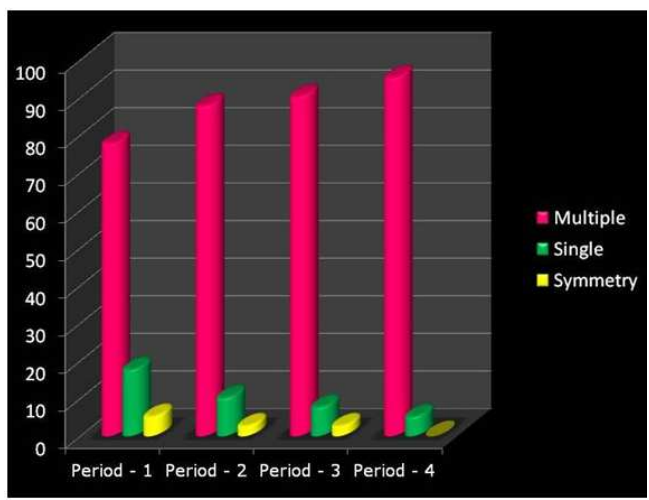

Graph 1. Comparison of pelvic malalignment of Soccer players from period - 1 to period - 4 
Table 18. Total analysis of observations

\begin{tabular}{ll}
\hline Malalignment & Percentage (\%) \\
\hline Multiple (35) & 87.5 \\
Single (04) & 10 \\
Symmetry Pelvis (01) & 2.5 \\
\hline
\end{tabular}

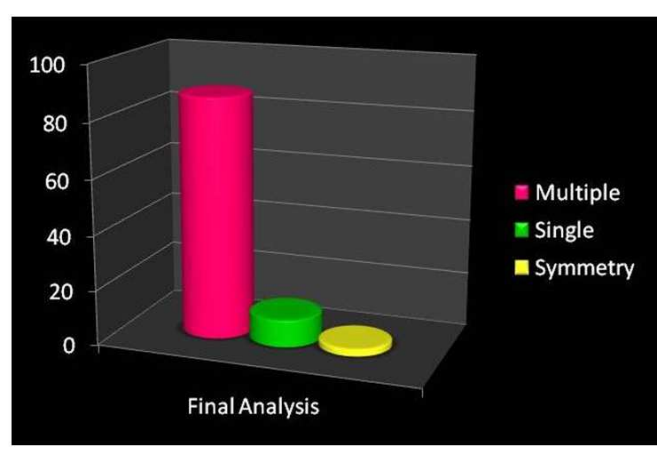

Graph 2. Final analysis of the observations

\section{Discussion}

The pelvic malalignment syndrome is the most common in soccer players. An understanding of this 'malalignment syndrome' requires knowledge of the common presentations of malalignment and the techniques used to diagnose and treat.

According to the e-source on pelvic evaluation (2010/09), there are four common presentations on innominate malalignment but the current study observed that there are seven possible innominate malalignment presentations. And the possible three sacral stucks for professional soccer players are also observed in the present study which was not mentioned in the previous studies (Pelvic evaluation, 2010; Abdolhamid Daneshjoo, 2013; Fernando Idoate, 2011; Julie Hides, 2012).

The present study shows that Anterior and Posterior Innominate stuck can occur due to the changes in the strength of Quadriceps and Hamstring muscles which is similar to the previous work done by Abdolhamid,2013.

According to Abdolhamid,2013, the physical performance and movement patterns of Soccer players may change the balance of strength in both the legs, not particularly to the dominant kicking leg which is contradict to the current study in which we identified the muscle imbalance and malalignment is more common in preferred kicking leg.

Rectus abdominis undergoes hypertrophy in the soccer players which is a common observation between the current study and Fernando, 2011.In the present study we observed that change in the strength and bulk of Rectus abdominis in the preferred kicking leg results in posterior innominate stuck in the soccer players but Fernando, 2011 stated that changes in the strength and bulk of rectus abdominis is bilateral and asymmetrical.

Julie hides, 2012 stated that the imbalance between Multifidus and Erector spinae results in Lumbo-pelvic instability and this adds an additional proof to the reason for rotational stuck of sacrum in our current study. In the present study ,some additional observations has been made regarding the sacral stuck, bilateral tightness of Multifidus and Erector spinae results in Counter-Nutational stuck of sacrum and bilateral weakness of the above mentioned muscles results in Nutational stuck of sacrum.

The above mentioned facts, observations and results indicated that an imbalance in the key muscles around the bony pelvis will affect the Sacro-pelvic stability.

\section{Conclusion}

The present study is mainly focused on the common presentations and manual diagnostic techniques for pelvic malalignment syndrome. We used simple bony palpation method to assess the various pelvic malalignments in football players. While there are many methods to determine pelvic symmetry or asymmetry, this method is very simple one and that is consistently works well for me. Our method of assessing the pelvic malalignments, even works well to the common individuals suffering with low back $\&$ pelvis pain or dysfunction due to various pelvic malalignment presentation. To be masterly skilled in our current techniques of evaluating the pelvic malalignment it is very simple and essential that you will need to be able to locate the anterior superior iliac spines (ASIS) and posterior superior iliac spines (PSIS), able to judge the levelness of both iliac crests when viewing from the front or rear even in different postures like standing, lying in supine \& prone also while performing the movements during the assessment.

The repeated measurement, and analysis proves that the "asymmetry of pelvis" which may be "ipsilateral" or "contralateral" to the preferred kicking leg. The results showed an imbalance of the key muscles associated with Sacro-pelvic stability. In this study, we observed, $87.5 \%$ i.e., the majority of the soccer's are suffering with multiple pelvic malalignments, includes innominate \& sacral stuck. The soccer's suffering with single component malalignment account for about $10 \%$, includes innominate or sacral stuck and soccer's who had the symmetry pelvis is only $2.5 \%$. Noted, the pelvic rotations are relative and it is not always necessary to know which side is the dysfunctional side if you are treating them, as you should treat both sides of the pelvis.

To justify our diagnosis we access the muscles of the functional slings and the muscles around the bony pelvis related to the malalignment of the pelvic unit. The techniques were repeated in different position and also performed for two times per day and continued for fifteen days to standardize.

However, This method of assessing the pelvic malalignments until then not documented. In this sense, the current study is mainly focused on the analysis and documentation of the different common presentations in pelvic malalignments which are common in soccer players and its simple method of evaluation is the literature state of the art. The present study may provide useful information to analyze common presentations of pelvic malalignments in different sports. 


\section{References}

[1] Abdolhamid Daneshjoo et al., Bilateral and Unilateral Asymmetries of Isokinetic strength and Flexibility in Male Young Professional Soccer Players. Journal of Human Kinetics.36, 45-53. 2013.

[2] Fernando Idoate et al., Soccer attenuates the Asymmetry of Rectus abdominis Muscle observed in Non- Athletes. PLOS ONE . 6(4), 1-7. 2011.

[3] F.P.Wedel,D.O., Evaluation and Treatment of Sacral Somatic
Dysfunction.Instruction manuals published by Michel Bakker. 2013.

[4] Julie Hides \& Warren Stanton., Muscle imbalance among Elite Australian Rules Football Players, A longitudinal study of changes in trunk muscle size. Journal of Athletic training.47(3), $314-319.2012$.

[5] Liz Gaggini,M.A.., The asymmetric pelvis. International Association of Structural Integrators. 1-7. 2010

[6] Pelvic Evaluation., www.waltfritz seminars.com/myofascial resource/wp-content/uploads/2010/09. 